\title{
Modeling SWCNT Band-gap and Effective Mass Variation using a Monte Carlo Approach
}

\author{
Karim El Shabrawy, Student Member, IEEE, Koushik Maharatna, Member, IEEE, Darren Bagnall, \\ Member, IEEE, Bashir Al-Hashimi, Fellow, IEEE
}

\begin{abstract}
Synthesizing Single-Walled Carbon-Nanotubes (SWCNTs) with accurate structural control has been widely acknowledged as an exceedingly complex task culminating in the realization of CNT devices with uncertain electronic behavior. In this paper we apply a statistical approach in predicting the SWCNT band-gap and effective mass variation for typical uncertainties associated with the geometrical structure. This is firstly carried out by proposing a simulation-efficient analytical model that evaluates the band-gap ( $\mathrm{Eg}$ ) of an isolated SWCNT with a specified diameter $(d)$ and chirality $(\theta)$. Similarly, we develop a SWCNT effective mass model, which is applicable to CNTs of any chirality and diameters $>1 \mathrm{~nm}$. A Monte Carlo method is later adopted to simulate the band-gap and effective mass variation for a selection of structural parameter distributions. As a result, we establish analytical expressions that separately specify the band-gap and effective mass variability $\left(E g_{\sigma}, m_{\sigma}{ }_{\sigma}\right)$ with respect to the CNT mean diameter $\left(d_{\mu}\right)$ and standard deviation $\left(d_{\sigma}\right)$. These expressions offer insight from a theoretical perspective on the optimization of diameter-related process parameters with the aim of suppressing band-gap and effective mass variation.
\end{abstract}

Index Terms-Single Walled Carbon Nanotube (SWCNT), Third-Nearest-Neighbor Tight-Binding (TB) model, Band-gap variation, Effective mass variation, CNT device models.

\section{INTRODUCTION}

$\mathrm{C}$ ARBON NANOTUBES (CNTs) possess distinctive electronic properties that make them ideal candidates for next generation on-chip devices and interconnects [1-4]. Unlike other nanoscale materials, they can remarkably exhibit semiconducting $(E g>0)$ or metallic $(E g=0)$ behavior depending on their geometrical structure, which consists of the diameter $(d)$ and chirality $(\theta)$ [5]. At present, the fabrication of CNTs with accurate diameter and chirality control is a serious challenge and as a result a large band-gap $(E g)$ variability is

Manuscript received December 2, 2008; revised March 26 2009. A preliminary version of this work has been presented at ICICDT '08 [36]. The work presented in this paper includes the following extensions: A) Enhanced model in distinguishing metallic and semiconducting tubes B) Generation of new band-gap and effective mass variation models using a Monte Carlo approach. All authors are with the School of Electronics \& Computer Science, University of Southampton, SO17 1BJ, UK (e-mail: kes06r@ecs.soton.ac.uk). Copyright ( 2009 IEEE. Personal use of this material is permitted. However, permission to use this material for any other purposes must be obtained from the IEEE by sending a request to pubs-permissions@ieee.org. typically observed for a set of CNTs grown under identical process conditions $[3,6,7]$. Equally susceptible to structural variation is the effective mass $\left(m^{*}\right)$, which has a crucial influence upon the CNT carrier transport properties [8,9]. Consequently, due to the unpredictability associated with these parameters, the implementation of CNT based devices with desired performance characteristics has been problematic [3].

In an attempt to tackle this issue, researchers have proposed the adoption of statistical process optimization techniques to optimize the CNT growth process and generate narrowly distributed geometrical characteristics around a desired mean value $[6,7,10-15]$. Progress, however, has been limited due to the incomplete understanding associated with the CNT growth mechanism [16]. Further, the impact of CNT geometrical structure on the performance characteristics of various CNT Field-Effect-Transistors (FETs) has been experimentally [3, $10,17]$ and theoretically [18-22] examined. Yet, no statistical model defining the CNT band-gap and effective mass distributions has evolved from these studies, especially with regards to semiconducting SWCNTs.

Here, we present a statistically supported model that predicts the CNT band-gap and effective mass distribution properties for a given structural variation. The structural dispersions considered reflect typical spreads identified in CNT geometry after Chemical Vapor Deposition (CVD) synthesis. As an outcome of the models produced, we offer better insight on ways in which CVD process parameters such as the mean CNT diameter $d_{\mu}$ and standard deviation $d_{\sigma}$ could be optimized to achieve a required band-gap or effective mass variation. Moreover, the proposed models could be incorporated into compact device models to accurately simulate a substantial number of dissimilar CNT-devices over a judicious time period.

The rest of this paper is structured as follows: Section II provides a brief review of SWCNT electronic properties and discusses the shortcomings of present band-gap models. In Section III we develop an analytical model that predicts the band-gap of an isolated SWCNT for a given diameter and chirality. Section IV proposes a CNT effective mass model that takes account of all chiralities and diameters $>1 \mathrm{~nm}$. Section V presents a set of band-gap distributions generated using a Monte Carlo approach for both Gaussian and Gamma dispersed diameters. These results are exploited with the aim of creating analytical models relating band-gap variation to the 
corresponding structural distribution properties. Section VI follows a similar procedure to that taken in Section $\mathrm{V}$ where the effective mass variation is articulated with respect to the Normally distributed diameter properties. The implications of the proposed models are assessed and summarized in Section VII.

\section{REVIEW OF SWCNT ELECTRONIC PROPERTIES}

A Single-Walled Carbon Nanotube (SWCNT) is a selfassembled hollow cylinder constructed from a rolled-up sheet of graphene [23]. The tube can be uniquely defined by a rollup vector known as the chiral vector, $\mathbf{C}_{\mathbf{h}}$, which can be expressed in terms of the primitive unit vectors $\mathbf{a}_{1}$ and $\mathbf{a}_{2}$ of the graphene lattice [24]:

$$
\mathbf{C}_{\mathbf{h}}=m \mathbf{a}_{1}+n \mathbf{a}_{2}
$$

where $m$ and $n$ are integers that are specific to a $(m, n) \mathrm{CNT}$ [24]. The magnitude of $\mathbf{C}_{\mathbf{h}}$ corresponds to the circumference around the nanotube. This can be used to determine the diameter $(d)$ as denoted by (2).

$d=\frac{\sqrt{3}}{\pi} a_{c c} \sqrt{m^{2}+n m+n^{2}}$

In (2) the constant $a_{c c}(0.142 \mathrm{~nm})$ represents the nearestneighbor C-C distance [25]. The chiral angle $(\theta)$ is defined as the angle between $\mathbf{C}_{\mathbf{h}}$ and $\mathbf{a}_{\mathbf{1}}$, which can be expressed as [26]:

$\theta=\tan ^{-1}\left(\frac{\sqrt{3} n}{2 m+n}\right)$

Previous studies have confirmed the sensitivity of the CNT's band-gap to both $d$ and $\theta$, where several theoretical and analytical models have been established to simulate their electronic band-structure $[23,24,26-30]$. Amongst the commonly used band models are the nearest-neighbor TightBinding (TB) approach with zone-folding [23, 24, 26-28], the Extended Hückel Theoretical (EHT) technique [29] and Firstprinciple ab-initio calculations [23, 30]. Although very accurate, the specified models are computationally intensive and possess a time complexity that increases with the size of the nanotube. For applications that require the simultaneous simulation of millions of distinct CNT-devices these models would be very difficult to utilize in a timely manner.

Analytical models such as those mentioned in [25, 31] were formed by experimentally probing a small number of semiconducting CNTs in order to measure their geometric structure and corresponding electrical output characteristics. In turn, results were plotted and curves extrapolated to acquire the CNT band-gap [30]. Not only were these results based on undersized samples of CNTs but the measurements were found to be strongly dependent upon the characterization technique employed [7, 32, 33]. Moreover, it has been argued that the statistical validity of measurements made using bulk sensitive probes is uncertain due to the lack of sensitivity in the characterization of individual nanotubes present within a given sample [7, 11]. Although the analytical model proposed in [34] does consider chirality, they presume that the type of CNT being dealt with is already known.

\section{MODELING BAND-GAP OF ISOLATED SWCNT}

In this section, we propose an analytical model with a time complexity that is independent of the size of the nanotube and simply consists of a single expression that directly determines the band-gap of an isolated SWCNT.

Normally, the electronic properties of a SWCNT are obtained by firstly computing the dispersion relation of graphene using a TB approach. Next, the Born-von Karman periodic boundary conditions are imposed along the circumferential direction slicing the $2 \mathrm{D}$ band-structure of graphene into 1D sub-bands [23]. If one of these slices intersects with a high symmetry $\mathrm{K}$ point in the Brillouin zone of graphene (where conduction and valence band touch at the Fermi-level) the nanotube can be considered metallic [35]. Otherwise, the nanotube is semiconducting with a finite $E g$. According to the choice of $n$ and $m$, each CNT will have a different electronic band-structure. This is known as the zonefolding technique and is sufficient in approximating the CNT band-gap for our study.

However, in $[28,32]$ it was shown that the nearest-neighbor TB approximation does not accurately reproduce the graphene band-structure when compared to ab initio calculations. Instead, it was revealed that the third-nearest-neighbor TB approach yielded better fitting results along the high-symmetry points of the Brillouin zone and that CNT band-structure models improved by the inclusion of more distant neighbors [28].

Using the third-nearest-neighbor TB approach with fitting parameters extracted from [28] in conjunction with the zonefolding technique we carried out a number of simulations in

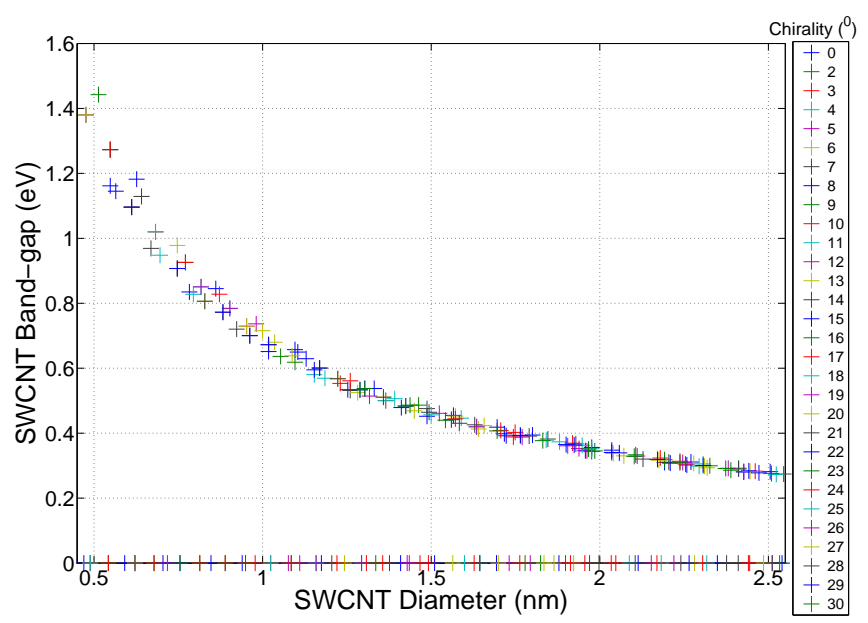

Fig. 1 SWCNT band-gap $\left(E_{g}\right)$ for different geometrical properties, $d$ and $\theta$. CNTs with different chirality are illustrated by separate colour shading. 
MATLAB where the band-gap was calculated for a set of 286 SWCNTs. These tubes were characterized by all possible chiralities, $0^{0}-30^{\circ}$, and diameters ranging between 0.45 $2.55 \mathrm{~nm}$. Fig. 1 shows the calculated $E g$ for various $\theta$ and $d$.

\section{A. Distinguishing semiconducting and metallic SWCNTs}

The geometric variables $(\theta, d)$ that produced a zero band-gap and non-zero band-gap were separately plotted as data points, indicated by symbols + and $\circ$ respectively, in the structural parameter space shown in Fig. 2.

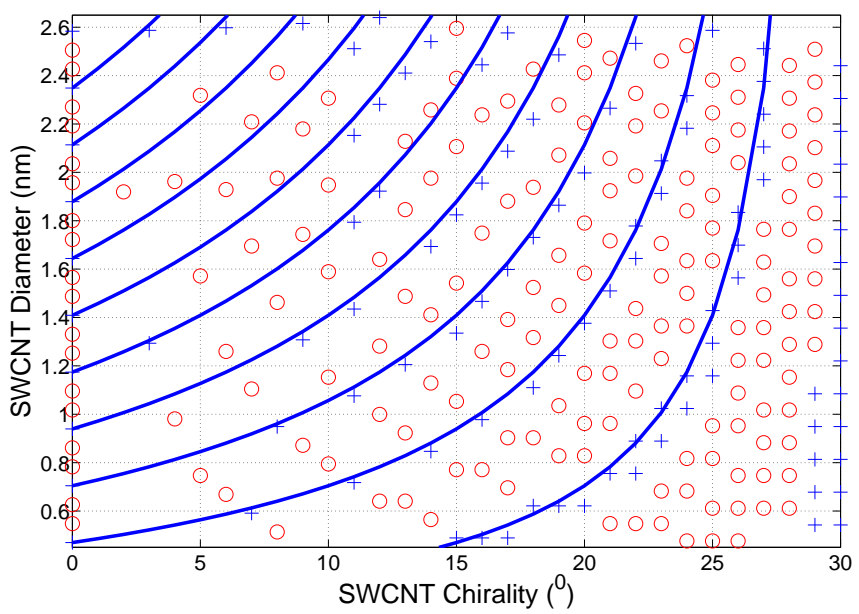

Fig. 2. CNT structural parameter space indicating data points $(\theta, d)$ for metallic and semiconducting tubes. Curves represent equation (5) for $p=1-10$.

In order to establish an expression that will predict the zero band-gap data points, a relationship between $d$ and $\theta$ was initially derived as follows. By rearranging (3) and substituting into (2) gives the following:

$d=\frac{3 a_{c c}}{2 \pi} \frac{n}{\sin \theta}$

Assuming that the sine function of (4) can be approximated by a first-degree polynomial $(b \theta+c)$ over the interval $0^{0} \leq \theta$ $\leq 30^{\circ}$ then it can be deduced that the diameter $d$ is inversely proportional to $b \theta+c$. This assumption is based on the $0.6 \%$ normalized root-mean-square (NRMS) error achieved when approximating a sine function with a linear polynomial over the required interval.

When examining the zero band-gap data points in Fig. 2 it could be seen that if several distinct curves were to be fitted over the points then they would all share the same asymptote at $\theta=30^{\circ}$. Thus, $b$ can be expressed in terms of $c$ as $b=-c / 30$, leaving us only with one unknown variable; $c$.

Upon close inspection of the zigzag tubes $\left(\theta=0^{0}\right)$ we detect a pattern in the tube diameters that offer a zero band-gap. These diameters include the third multiple of the smallest possible diameter. In theory, the smallest possible SWCNT diameter can be determined from (2) given a tube indices of $(1,0)$, providing $d_{\min }=\sqrt{3} a_{c d} / \pi$. Therefore, the value of $c$ can be expressed as a reciprocal of $3 p d_{\min }$, where $p$ is a positive integer. Combining the above mentioned results allows us to form an expression for the zero band-gap data points:

$D(\theta, p)=\left(\frac{\pi}{3 p \sqrt{3} a_{c c}}\left(1-\frac{\theta}{30}\right)\right)^{-1}$

Equation (5) represents the zero band-gap curves shown in Fig. 2 where $p$ selects the curve of interest. However, (5) is unable to predict Armchair tubes $\left(\theta=30^{\circ}\right)$ as well as zero diameter tubes. Thus, to cover all zero band-gap data points in Fig. 2, a function can be formulated that assumes a value of zero only when either:

- the diameter $d$ lies on a curve approximated by (5) for a given geometry,

- or, the SWCNT is an Armchair $\left(\theta=30^{\circ}\right)$ tube,

- or, the SWCNT has a null diameter $(d=0 \mathrm{~nm})$.

Quantitatively, this can be expressed as:

$f(\theta, d)=d(30-\theta) \prod_{p=1}^{N}(D(\theta, p)-d)$

Where $N$ represents the total number of curves taken into consideration. $f(\theta, d)$ can be rearranged giving:

$f(\theta, d)=d(30-\theta) \prod_{p=1}^{N}\left(1-\frac{\alpha}{p}\right) \frac{p d}{\alpha}$

Where:

$\alpha=\frac{d \pi}{90 \sqrt{3} a_{c c}}(30-\theta)$

To solve (7), $N$ iterations are required where each product term is evaluated individually and then multiplied by the rest of the function. This process is time consuming for simulating a design with multiple CNT-devices and hence there is a need to reduce the product term to a non-iterative form. Moreover, the accuracy of (7) is limited by the number of curves chosen when defining $N$ since the more curves considered the higher the number of zero band-gap data points covered. One way of resolving these issues is to consider a high number of curves and taking the limit as $N$ tends towards a finitely large value. To accomplish this, we firstly multiply and divide (7) by the conjugate of the product term. Then, $f(\theta, d)$ is rearranged to:

$$
f(\theta, d)=\frac{\alpha 90 \sqrt{3} a_{c c}}{\pi} \prod_{p=1}^{N}\left(1-\frac{\alpha^{2}}{p^{2}}\right) \prod_{p=1}^{N}\left(\frac{p^{2} d}{\alpha(p+\alpha)}\right)
$$


As $N$ tends towards a finitely large value, the first product term of (9) could be approximated by a $\operatorname{sinc}(\pi \alpha)$ function and the second product term tends towards a high value. Since only the roots of $f(\theta, d)$ are of interest we can approximate (9) as:

$$
f(\theta, d)=|\Re(\sin (\pi \alpha))|
$$

Where $\mathcal{R}$ is a function that rounds values to the nearest integer, giving $f(\theta, d)=0$ for metallic CNTs and $f(\theta, d)=1$ for semiconducting tubes.

Equation (10) was evaluated by sourcing the geometrical properties of all 286 randomly distinct metallic and semiconducting CNTs. The results for $f(\theta, d)$ are depicted in the structural parameter space of Fig. 3.

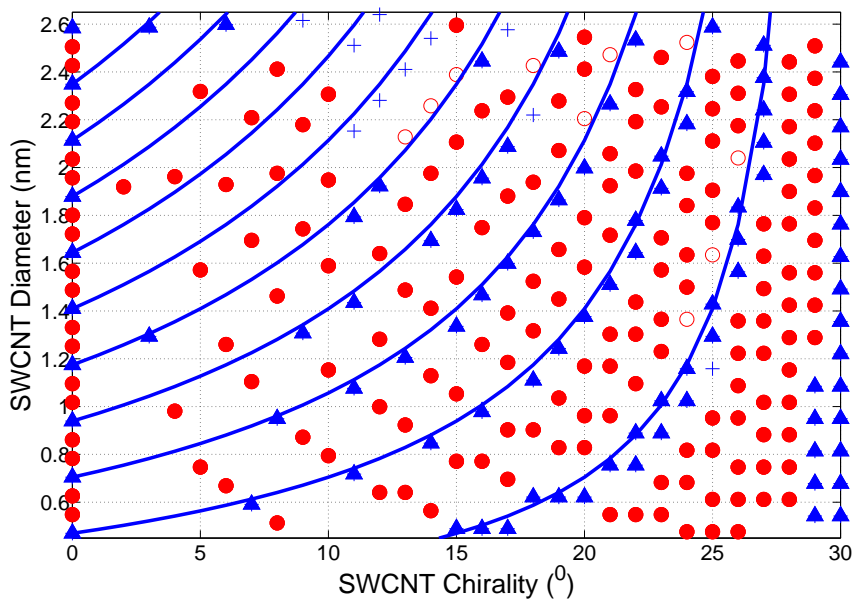

Fig. 3. CNT structural parameter space indicating data points $(\theta, d)$ for metallic and semiconducting tubes. $+(\mathrm{o})$ represents metallic (semiconducting) results obtained using the third-nearest-neighbor TB approach with the zone-folding technique. $\boldsymbol{\Delta}(\bullet)$ represents metallic (semiconducting) tubes identified correctly by equation (10).

From Fig. 3 we found that 266 out of a total of 286 tubes are correctly assigned. That is, the formulated expression, (10), can correctly distinguish metallic and semiconducting tubes with $93 \%$ accuracy when compared to the third-nearestneighbor TB approach with zone-folding. This value offers an $11 \%$ improvement over the accuracy obtained in [36].

\section{B. Calculating band-gap of semiconducting SWCNTs}

It is clear from Fig. 1 that for all semiconducting SWCNTs the dependence of $E g$ on diameter is inversely proportional. This reinforces the $1 / d$ relationship derived in $[1,23,25,27,30,31$, $34,37]$. However, all these sources differ on an additional factor that is used during the calculation of a semiconducting CNT band-gap; the overlap energy $\gamma_{0} \cdot \gamma_{0}$ is a constant that has been debated to be in the range $2.45-2.90 \mathrm{eV}$ and no agreed value has ever emerged [34]. In [34] it was mentioned that the reason for the resulting discrepancy in $\gamma_{0}$ is due to the fact that chirality is neglected when interpreting the band-gap for different diameters. Thus, here we have plotted $E g$ with respect to $d$ and $\theta$ for the semiconducting nanotubes only. Subsequently, a curve-fitting technique is used to establish a relationship between $E g, d$ and $\theta$. Fig. 4 shows the resulting optimal curve given by:

$E g=10.2 \frac{3 a_{c c}}{2 \pi d}=\frac{0.692}{d}$

Equation (11) confirms that $E g$ is proportional to $1 / d$ and the constant of proportionality is independent of $\theta$. Moreover, a value of $2.44 \mathrm{eV}$ is calculated from (11) for the overlap energy constant, which is in close agreement with that derived using First-principles $(2.5 \mathrm{eV})$ in [23]. This offers a consistency check for our approach.

When the semiconducting CNT band-gap values were generated using (11) and compared against the third-nearestneighbor TB approach with zone folding, this yielded a NRMS error of $1.75 \%$ only.

To further verify (11), comparisons were made with respect to two references that experimentally analyzed semiconducting SWCNTs using different techniques. In [25] Scanning Tunneling Spectroscopy (STS) was used to examine the electronic properties as a function of $d$ and $\theta$ for a set of SWCNTs. The measurements obtained are clearly indicated in Fig. 4. In [31] Scanning Tunneling Microscopy (STM) characterization of the SWCNTs was undertaken and the bandgap of 5 nanotubes were extracted and recorded as shown in Fig. 4. Through inspecting the experimental data points we can validate that the measured band-gap values lie very close to the curve proposed by (11). Additionally, in [31], the overlap energy was approximated as $2.45 \mathrm{eV}$, which matches well with our result.

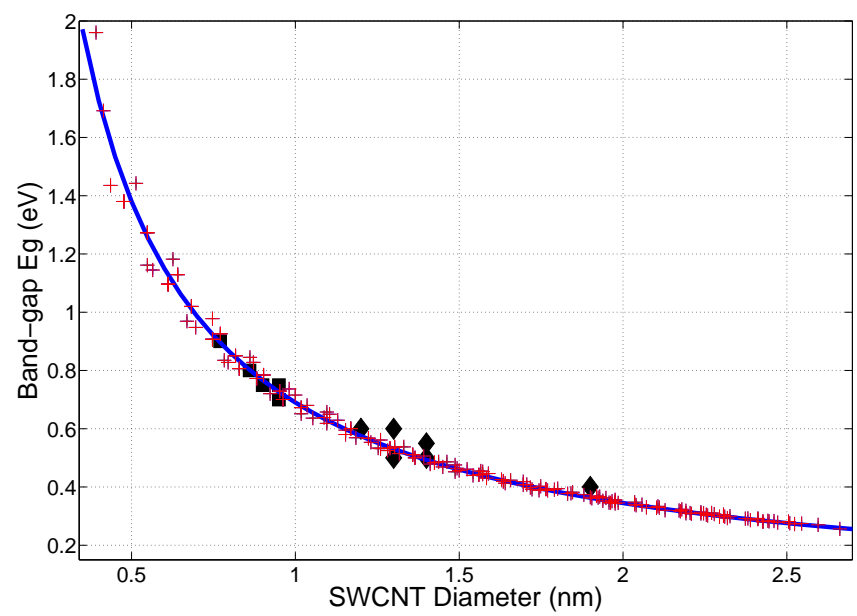

Fig. 4. Semiconducting band-gap vs. diameter for all $\theta .+$ represents results obtained using the third-nearest-neighbor TB approach with the zone-folding technique. - is the fitting curve given by equation (11). and a depict experimental measurements made in [25] and [31], respectively.

Equation (12) represents a simplistic model that unifies the key expressions established thus far. Given any SWCNT diameter $(\mathrm{nm})$ and chirality $\left({ }^{0}\right),(12)$ computes the SWCNT 
band-gap with a runtime independent of tube size and the value of $N$. This is more simulation efficient than the model proposed in [36], which possesses a time complexity of the order of $O(N)$.

$$
E g=|\Re(\sin (\pi \alpha))| \frac{0.692}{d}
$$

\section{Modeling EFFECTIVE Mass of ISOlATED SWCNT}

The electron and hole effective mass is an extensively used parameter for modeling electrical transport in semiconductor devices [8, 38]. It is inversely proportional to the carrier mobility and can therefore be utilized in characterizing the CNT's electric conductivity [8].

Typically, the effective mass $m^{*}$ of a semiconducting material is determined from the curvature of the corresponding band-structure [8]. Formally, this is expressed as:

$$
m^{*}=\hbar^{2}\left(\frac{\partial^{2} E}{\partial k^{2}}\right)^{-1}
$$

where $m^{*}$ is usually represented in terms of the electron rest mass $m_{0}[8]$.

In our study we only consider the first conduction/valence bands as they are the dominant sub-bands participating in CNT carrier transport [22]. Also, due to the close symmetry between the CNT conduction and valence band we can assume that the hole effective mass is approximately the same as the electron effective mass $[8,9]$. Thirdly, the bottom of the conduction band could be estimated using a second order polynomial fit (e.g. $E(k)=A k^{2}+B k+C$ ), which can be substituted into (13) to obtain the curvature. This is known as the parabolic effective mass model (EFM) and has demonstrated reasonable accuracy for CNT diameters greater than $1 \mathrm{~nm}[8,22]$. At lower diameters the parabolic EFM becomes imprecise due to the curvature effect [8].

Again, using the third-nearest-neighbor tight-binding technique with zone folding we calculate the band structure for 133 semiconducting nanotubes having diameters and chiralities ranging between $1-2.6 \mathrm{~nm}$ and $0^{0}-30^{\circ}$, respectively. Next, the parabolic EFM is employed to realize the effective mass. These results are illustrated in Fig. 5.

Fig. 5 shows that the effective mass has a dependency on chirality and generally decreases with diameter, which is in agreement with $[8,9,22,38]$. In comparison to $a b$ initio calculations made in [9] for chiral and achiral CNTs we also find that the data points do not fall on a simple trend line. In addition, at similar diameters, we confirm that the effective mass of chiral tubes is higher than achiral tubes [9].

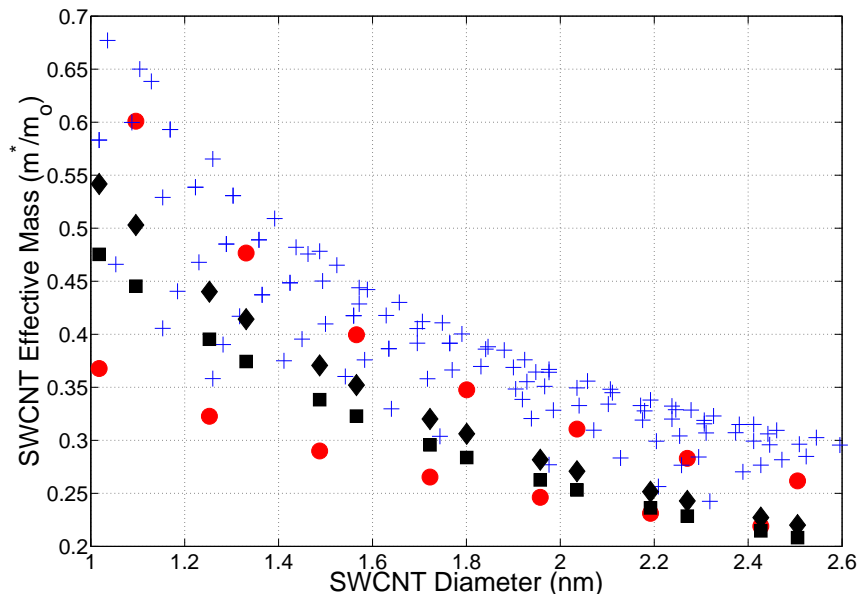

Fig. 5. SWCNT effective mass of the lowest conduction sub-band for different geometrical properties, $d$ and $\theta$. $\bullet$ and + represent achiral and chiral CNTs, respectively. and a depict effective mass models proposed for zigzag tubes in [22] and [38], respectively, with $\gamma_{0}=2.7 \mathrm{eV}$.

Previously proposed effective mass models such as in [22] and [38] (shown in Fig. 5) were analytically derived for zigzag tubes only. Here, we employ a regression technique Response Surface Modeling (RSM) - to predict the effective mass for all tube chiralities within 1-2.6nm. The RSM method renders a first-order model relating the predictors $(\theta, \log (d))$ to the response variable $\left(\log \left(m^{*}\right)\right)$. After performing an inverselogarithmic transform, the model yields the following:

$m^{*}=\frac{8 m_{0} \hbar^{2}}{9 \gamma_{0}^{2} a_{c c}} d^{-0.7835} e^{7.4 \times 10^{-3} \theta}$

where $\gamma_{0}(2.44 \mathrm{eV})$ is the overlap energy calculated in the prior Section. (14) was found to predict the effective mass data points of Fig. 5 with a NRMS error of $9.7 \%$. This is a good approximation given that there is a nonlinear correlation between the effective mass and chirality.

\section{SIMULATING \& MODELING SWCNT BAND-GAP VARIATION}

The following section initially defines the probability functions chosen to replicate a realistic spread in SWCNT diameter and chirality. By varying the distribution properties and running a set of Monte Carlo simulations we generate the corresponding band-gap dispersions, which are subsequently compared and statistically analyzed. As an outcome, simplistic yet accurate models are proposed providing a relationship between the semiconducting CNT diameter distribution properties and the resulting band-gap variation characteristics; $E g_{\mu}$ and $E g_{\sigma}$.

\section{A. SWCNT diameter distribution}

The SWCNT structural distribution properties reported todate differ considerably due to the utilization of alternative synthesis techniques and growth conditions $[4,6,7,11-15,33$, 
39].

As the Chemical Vapor Deposition (CVD) synthesis process appears to be most promising in terms of scalability for CNT production $[6,13]$, we have deliberately restricted our survey of CNT structural data to references employing the CVD technique.

Recently, in [13] it was proposed that at any given CVD process condition, so long as the carbon feeding rate is fixed, there is an optimal diameter of nanoparticles that nucleate nanotubes. Thus, assuming that the process of defining the catalyst particle size can be optimized to give a narrow distribution around a specified $d_{\mu}$, we can expect that as the number of fabricated SWCNTs increases significantly for a given batch, the spread in diameter will converge towards a Gaussian distribution. Furthermore, having synthesized and measured the diameter distribution in a sizable mixture of CNTs, it has been found that most groups use a Gaussian fit for their diameter profiles [7, 12-14].

It is also practical to consider a non-Gaussian distribution for the diameter variability as many underlying process characteristics could demonstrate abnormal behavior [40]. Given that the Gamma profile is always positive and has a wider asymmetry compared to the Normal function it may provide a valuable outlook on the implications of an asymmetric variation in the catalyst particle size.

\section{B. SWCNT chiral angle distribution}

Unlike the CNT diameter, controlling the chiral angle can be more intricate [19]. Regulating the CNT chiral angle entails the manipulation of the molecular assembly of the CNT [16]. This level of manipulation is unattainable using conventional CVD processes and consequently a homogeneous spread in chirality is commonly observed within a collection of synthesized CNTs [33, 41]. Hence, for our purposes it is reasonable to assume a uniform random distribution in the CNT chiral angle.

\section{Semiconducting SWCNT band-gap distribution}

Here, the variation in CNT band-gap is determined by executing expression (12) over a large number of samples that are randomly generated from the selected structural distributions outlined above. A sample size of $1.5 \times 10^{5}$ nanotubes was chosen to gain adequate accuracy for the output variables $E g_{\mu}$ and $E g_{\sigma}$. This value was attained by progressively simulating larger sample sets and identifying the cut-off at which the output converges.

For each run of our Monte Carlo simulation the diameter distribution properties $d_{\mu}$ and $d_{\sigma}$ were varied between the ranges $1.01-1.71 \mathrm{~nm}$ and $0.04-0.2 \mathrm{~nm}$, respectively. Fig. 6 (a) shows a density estimation of a subset $\left(d_{\mu}=1.01 \mathrm{~nm}\right.$ and $d_{\sigma}=0.04-0.2 \mathrm{~nm}$ ) of semiconducting CNT band-gap results for a Gaussian spread in diameter. Fig. 6 (b) relates to a Gamma distributed diameter with equivalent properties. (a)

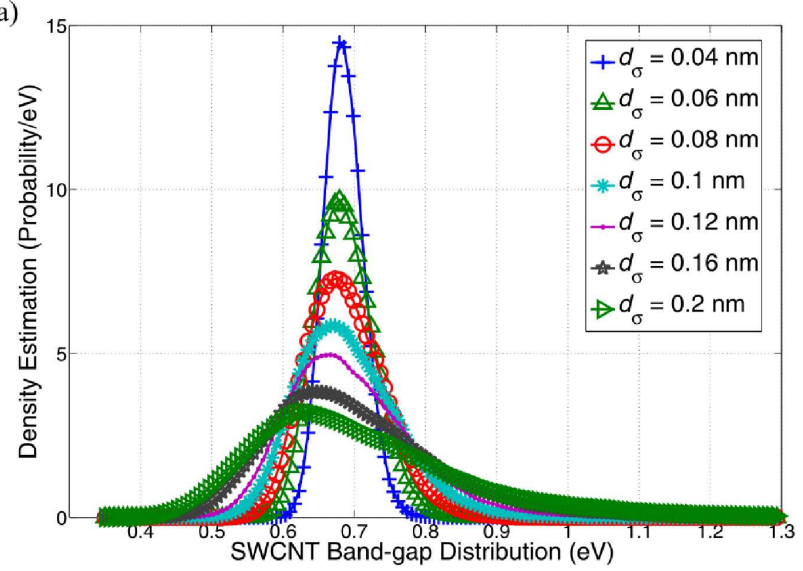

(b)

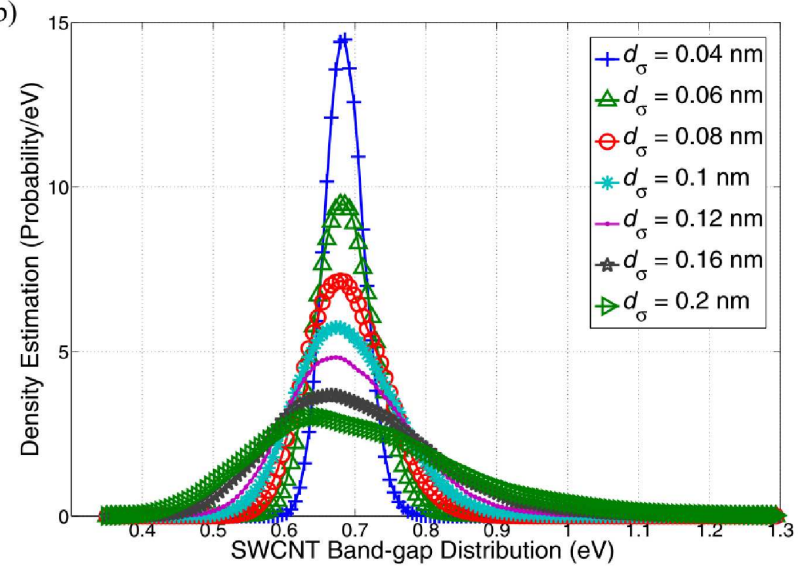

Fig. 6. Density estimation of semiconducting band-gap obtained from Monte Carlo simulations for a (a) Gaussian and (b) Gamma spread in diameter with $d_{\mu}=1.01 \mathrm{~nm}$ and $d_{\sigma}=0.04-0.2 \mathrm{~nm}$.

At first, it can be observed that the band-gap distributions generated for the Normally and Gamma dispersed diameters are very much indistinguishable. However, in comparison, the band-gap distributions for the Gamma spread in diameter were identified to be considerably less skewed. For instance, at $d_{\sigma}=$ $0.2 \mathrm{~nm}$ the skewness $(0.88)$ was estimated to be almost a third of the value calculated for the corresponding band-gap distribution with a Gaussian spread in diameter.

\section{Modeling SWCNT band-gap variation for Normally distributed diameters}

In realizing the mean $\left(E g_{\mu}\right)$ and variability $\left(E g_{\sigma}\right)$ of the produced band-gap dispersions we consider the sample mean and standard deviation, respectively, as unbiased estimators. This is possible due to the large sample sizes used in our Monte Carlo simulations.

When $E g_{\mu}$ was plotted against $d_{\mu}$ for band-gap dispersions generated from the normally spread diameter, as depicted by Fig. 7, it was found that the mean band-gap shifted upwards with higher $d_{\sigma}$ and even more so for smaller $d_{\mu}$. The optimal curves illustrated were created using the RSM regression technique that formed a second-order model given by (15). 


$$
\begin{aligned}
\log \left(E g_{\mu}\right)= & \beta_{0}+\beta_{1} \log \left(d_{\mu}\right)+\beta_{2} d_{\sigma}+\ldots \\
& \beta_{12} d_{\sigma} \log \left(d_{\mu}\right)+\ldots \\
& \beta_{11}\left(\log \left(d_{\mu}\right)\right)^{2}+\beta_{22} d_{\sigma}^{2}
\end{aligned}
$$

The regression coefficients of (15) are $\beta_{0}=-0.3727, \beta_{1}=-$ $0.9939, \beta_{2}=0.0852, \beta_{12}=-0.3456, \beta_{11}=0.0229$ and $\beta_{22}=$ 0.7070. Although (15) is highly accurate (NRMS error of $0.19 \%)$ within the mean diameter range specified in our simulation, (16) can also provide a reasonable approximation to (15), especially for large mean diameters.

$$
E g_{\mu}=\frac{0.692}{d_{\mu}}
$$

For $1.01 \mathrm{~nm} \leq d_{\mu} \leq 1.71 \mathrm{~nm}$ the NRMS error of (16) in predicting the actual mean band-gap is $2.7 \%$.

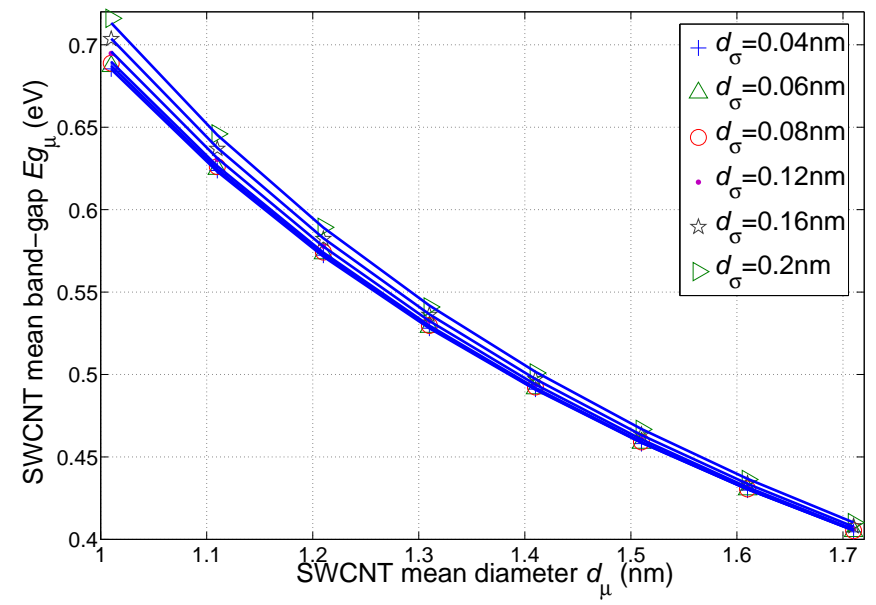

Fig. 7. SWCNT band-gap distribution mean $\left(E g_{\mu}\right)$ vs. mean diameter $\left(d_{\mu}\right)$ Data sets with different diameter standard-deviation $\left(d_{\sigma}\right)$ have been marked by a distinct symbol. - represents the fitted curve given by equation (15).

Next, the data points of the band-gap standard-deviation $\left(E g_{\sigma}\right)$ were plotted against the diameter standard-deviation $\left(d_{\sigma}\right)$ for different sets of $d_{\mu}$ as shown in Fig. 8.

Again, the curves of best fit were generated by employing the RSM method giving the following expression:

$$
\begin{aligned}
\log \left(E g_{\sigma}\right)= & \gamma_{0}+\gamma_{1} \log \left(d_{\sigma}\right)+\gamma_{2} \log \left(d_{\mu}\right)+\ldots \\
& \gamma_{12} \log \left(d_{\sigma}\right) \log \left(d_{\mu}\right)+\ldots \\
& \gamma_{11}\left(\log \left(d_{\sigma}\right)\right)^{2}+\gamma_{22}\left(\log \left(d_{\mu}\right)\right)^{2}
\end{aligned}
$$

where the regression coefficients are $\gamma_{0}=0.2883, \gamma_{1}=$ $1.4036, \gamma_{2}=-2.5146, \gamma_{12}=-0.1460, \gamma_{11}=0.0624$ and $\gamma_{22}=0.1515$.

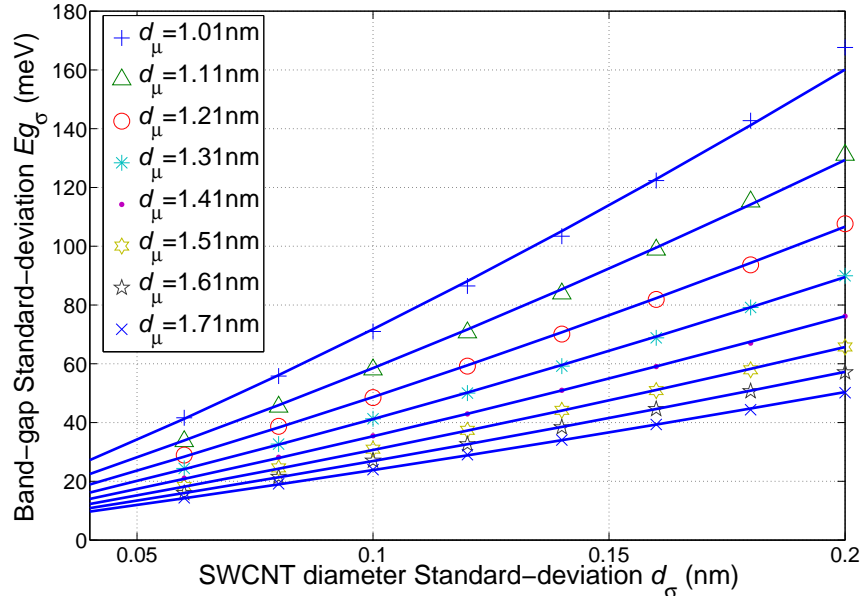

Fig. 8. Band-gap standard-deviation $\left(E g_{\sigma}\right)$ vs. diameter standard deviation $\left(d_{\sigma}\right)$. Data sets with different mean diameter $\left(d_{\mu}\right)$ have been marked by a distinct symbol. - represents the fitted curve given by equation (17) for each $d_{\mu}$.

The NRMS error of (17) in predicting the data points was evaluated as $0.66 \%$. Additionally, we can propose a more simplified approximation given by (18) that possesses a NRMS error of $2 \%$ within the ranges specified for $d_{\mu}$ and $d_{\sigma}$.

$E g_{\sigma}=\frac{0.8}{d_{\mu}^{2.2}} d_{\sigma}$

Equation (18) acknowledges a linear relationship between $E g_{\sigma}$ and $d_{\sigma}$. More interestingly, it indicates that the band-gap variation can be significantly reduced by defining nanoparticle sizes with a higher mean diameter.

\section{E. Modeling SWCNT band-gap variation for a Gamma distributed diameter}

The band-gap distribution properties $\left(E g_{\mu}, E g_{\sigma}\right.$. $)$ for the Gamma dispersed diameters were calculated in a similar manner to that outlined above.

As expected, it was firstly established that $E g_{\mu}$ could be accurately described by (15) and (16) with an NRMS error of $0.21 \%$ and $2.56 \%$, respectively. Subsequently, we found that the band-gap variation could also be expressed by (17) with regression coefficients $\gamma_{0}=-0.1019, \gamma_{1}=1.1624, \gamma_{2}=-2.195$, $\gamma_{12}=-0.0535, \gamma_{11}=0.0251$ and $\gamma_{22}=0.0583$. This model has a small NRMS error of $0.21 \%$. A more simplistic approximation of the variability is given by (19) where the NRMS error is only $0.8 \%$.

$E g_{\sigma}=\frac{0.73}{d_{\mu}^{2.1}} d_{\sigma}$

The close resemblance between (18) and (19) suggests that variation in CNT band-gap may not significantly depend on the underlying diameter distribution chosen within the ranges 
considered in our simulation. Equation (19) also reinforces the near inverse-square relationship between the mean diameter and band-gap variability.

Coincidently, the implication of increasing $d_{\mu}$ to reduce band-gap variation leads to the reduction of $E g_{\mu}$ as indicated by (14), which in turn yields CNT devices with improved performance characteristics such as higher mobility and low contact resistance [10, 42, 43]. Hence, according to our results, CNT synthesis processes have to be optimized in minimizing $d_{\sigma}$ as well as increasing $d_{\mu}$.

\section{Simulating \& Modeling SWCNT EFFECTIVE MaSS VARIATION FOR A GAUSSIAN DIAMETER DISTRIBUTION}

The effective mass dispersions were calculated by simulating (14) over $1.5 \times 10^{5}$ nanotube samples where the diameters were Normally distributed and the chiralities uniformly spread. As in the previous section, the same ranges for $d_{\mu}$ and $d_{\sigma}$ were also considered. Fig. 11 represents a subset of the CNT effective mass results showing a more positively skewed distribution with higher diameter variation.

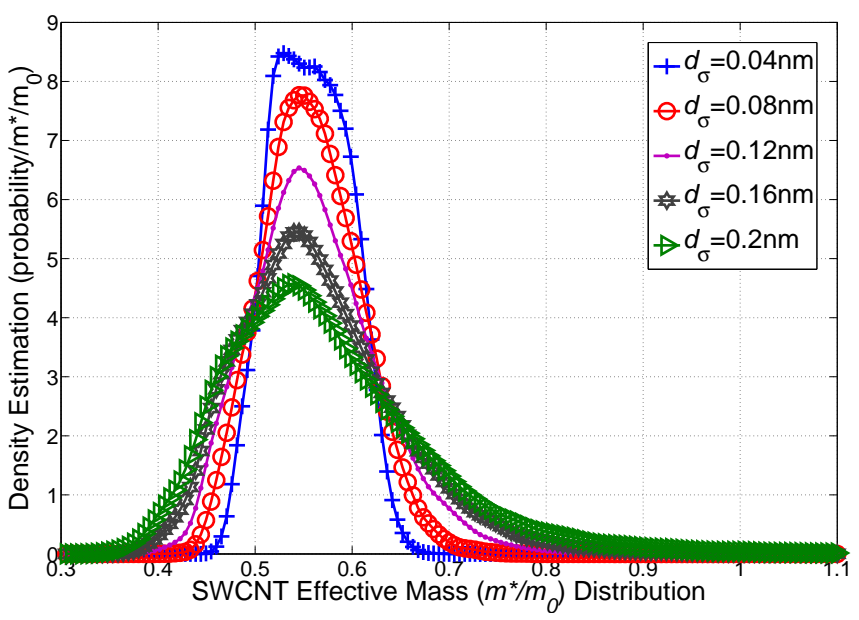

Fig. 9. Density estimation of effective mass obtained from Monte Carlo simulations for a Normally dispersed diameter with $d_{\mu}=1.01 \mathrm{~nm}$ and $d_{\sigma}=0.04$ $0.2 \mathrm{~nm}$.

The effective mass sample mean $\left(m_{\mu}^{*}\right)$ was evaluated for each $d_{\mu}$ and $d_{\sigma}$ as depicted in Fig. 10. Akin to the mean bandgap results, we observe that $m_{\mu}^{*}$ is somewhat shifted upwards with respect to the diameter variation, especially at lower mean diameters. The curves shown are given by:

$$
\begin{gathered}
\log \left(m_{\mu}^{*} / m_{0}\right)=\beta_{0}+\beta_{1} \log \left(d_{\mu}\right)+\beta_{2} d_{\sigma}+\ldots \\
\beta_{12} d_{\sigma} \log \left(d_{\mu}\right)+\ldots \\
\beta_{11}\left(\log \left(d_{\mu}\right)\right)^{2}+\beta_{22} d_{\sigma}^{2}
\end{gathered}
$$

where the regression coefficients are $\beta_{0}=-0.5823, \beta_{1}=-0.7804$, $\beta_{2}=0.0538, \beta_{12}=-0.2359, \beta_{11}=0.0176$ and $\beta_{22}=0.5039$. This model offers an NRMS error of $0.2 \%$.

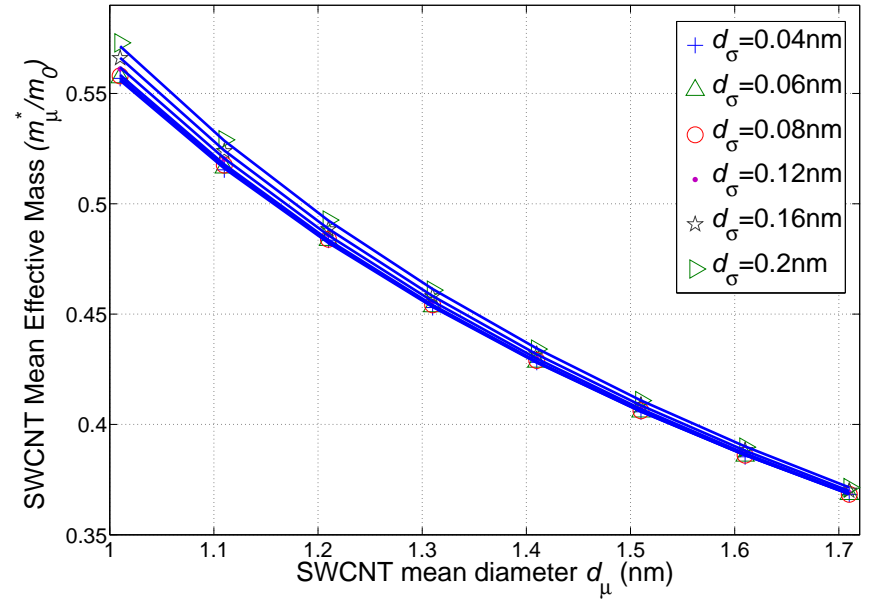

Fig. 10. SWCNT Effective Mass distribution mean $\left(m^{*} / m_{0}\right)$ vs. mean diameter $\left(d_{\mu}\right)$. Data sets with different diameter standard-deviation $\left(d_{\sigma}\right)$ have been marked by a distinct symbol. - represents the fitted curve given by equation (20)

We also propose a simplified expression for the mean effective mass, given by (21), which provides a slightly higher NRMS error of $3.0 \%$.

$m_{\mu}^{*}=\frac{0.56 m_{0}}{d_{\mu}^{0.8}}$

Lastly, a plot was created as shown in Fig. 11 to ascertain the variation in effective mass $\left(m_{\sigma}^{*}\right)$ with respect to the diameter standard-deviation for each $d_{\mu}$. The fitted curves illustrated are expressed by (22) with regression coefficients $\gamma_{0}=-3.4453, \gamma_{1}=5.6367, \gamma_{2}=-0.8807, \gamma_{12}=-5.276, \gamma_{11}=2.8868$ and $\gamma_{22}=0.2781$. (22) was estimated to predict the effective mass variation data points with an NRMS error of $0.48 \%$ only.

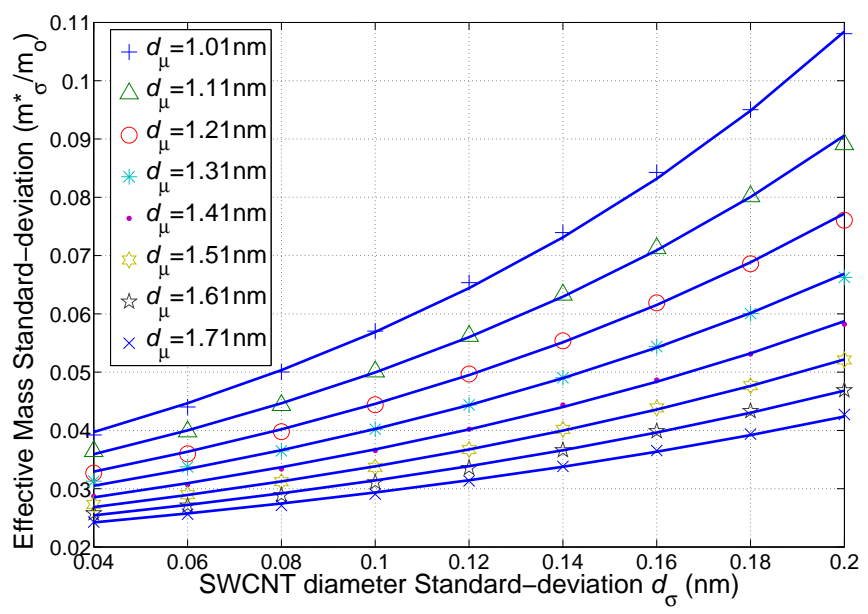

Fig. 11. Effective mass standard-deviation $\left(m^{*}{ }_{\sigma} / m_{0}\right)$ vs. diameter standard deviation $\left(d_{\sigma}\right)$. Data sets with different mean diameter $\left(d_{\mu}\right)$ have been marked by a distinct symbol. - represents the fitted curve given by equation (22) with new regression coefficients. 


$$
\begin{gathered}
\log \left(m_{\sigma}^{*} / m_{0}\right)=\gamma_{0}+\gamma_{1} d_{\sigma}+\gamma_{2} \log \left(d_{\mu}\right)+\ldots \\
\gamma_{12} d_{\sigma} \log \left(d_{\mu}\right)+\ldots \\
\gamma_{11} d_{\sigma}^{2}+\gamma_{22}\left(\log \left(d_{\mu}\right)\right)^{2}
\end{gathered}
$$

Once again we propose (23) as more concise form of the effective mass variability to that of (22). This comes with an extended NRMS error of $3.22 \%$.

$m_{\sigma}^{*}=\frac{0.037 m_{0}}{d_{\mu}^{1.36}} e^{4.78 d_{\sigma}}$

Unlike the band-gap variability, (23) indicates that the effective mass variation increases exponentially with diameter fluctuation. It is also observed that there is a reduction in $m^{*}{ }_{\sigma}$ with higher mean diameters, although not as much as with the band-gap variation.

\section{CONCLUSION}

In this paper we start by proposing analytical models for the CNT band gap and effective mass. These were derived from band-structures created using the third-nearest-neighbor TightBinding (TB) method in conjunction with the zone-folding technique. We demonstrate that the band-gap model accurately distinguishes $93 \%$ of a set of both metallic and semiconducting CNTs. In addition, the NRMS band-gap error recorded for the semiconducting tubes is only $1.75 \%$. The model is subsequently validated against two separate sources of experimental data.

We also propose a model with an NRMS error of $9.7 \%$ that predicts the effective mass of a semiconducting CNT possessing any chirality and diameter $>1 \mathrm{~nm}$.

In exploiting the models developed and running an extensive set of Monte Carlo simulations we established simulation-efficient and accurate models that predict the variation in CNT band-gap and effective mass for different structural distributions. The implications of our work advocate that CNT synthesis processes have to be optimized in minimizing the diameter variability $\left(d_{\sigma}\right)$ as well as increase the mean diameter $\left(d_{\mu}\right)$ to suppress band-gap and effective mass variations.

\section{ACKNOWLEDGMENT}

The authors would like to thank Dr. $\mathrm{CH}$ De Groot and Prof. Peter Ashburn from the University of Southampton, UK, for providing valuable feedback during their insightful discussions.

\section{REFERENCES}

[2] A. Nieuwoudt and Y. Massoud, "On the Impact of Process Variations for Carbon Nanotube Bundles for VLSI Interconnect," IEEE Transactions on Electron Devices, vol. 54, pp. 446-455, 2007.

[3] P. McEuen, M. Fuhrer, and H. Park, "Single-Walled Carbon Nanotube Electronics," IEEE Transactions on Nanotechnology, vol. 1, pp. 78-85, 2002.

[4] Y. Yao, Q. Li, J. Zhang, R. Liu, L. Jiao, Y. T. Zhu, and Z. Liu, "Temperature-mediated growth of single-walled carbon-nanotube intramolecular junction," Nature materials, vol. 6, pp. 283 - 286, 2007

[5] M. Terrones, "Science and Technology of the Twenty-First Century: Synthesis, Properties, and Applications of Carbon Nanotubes," Annual Review of Materials Research, vol. 33(1), pp. 419-501, 2003.

[6] D. Selbmann, B. Bendjemil, A. Leonhardt, T. Pichler, C.Taschner, and M. Ritschel, "A parametric study of the synthesis and purification of single-walled carbon nanotubes using the highpressure carbon monoxide process," Applied Physics A: Materials Science \& Processing, vol. 90, pp. 637-643, 2008.

[7] X. Liu, T. Pichler, M. Knupfer, M. S. Golden, J. Fink, H. Kataura, and Y. Achiba, "Detailed analysis of the mean diameter and diameter distribution of single-wall carbon nanotubes from their optical response," Physical Review B, vol. 66, 2002.

[8] S. Sreekala, X.-H. Peng, P. M. Ajayan, and S. K. Nayak, "Effect of strain on the band gap and effective mass of zigzag single-wall carbon nanotubes: First-principles density-functional calculations," Physical Review B (Condensed Matter and Materials Physics), vol. 77, p. 155434, 2008.

[9] G. L. Zhao, D. Bagayoko, and L. Yang, "Effective masses of charge carriers in selected symmorphic and nonsymmorphic carbon nanotubes," Phys. Rev. B, vol. 69, p. 2454162004.

[10] Y. Tseng, K. Phoa, D. Carlton, and J. Bokor, "Effect of Diameter Variation in a Large Set of Carbon Nanotube Transistor," Nano Letters, vol. 6, pp. 1364-1368, 2006.

[11] A. G. Nasibulin, P. V. Pikhitsa, H. Jiang, and E. I. Kauppinen, "Correlation between catalyst particle and single-walled carbon nanotube diameters," Carbon, vol. 43, pp. 2251-2257, 2005.

[12] T. Hiraoka, S. Bandow, H. Shinohara, and S. Iijima, "Control on the diamter of single-walled carbon nanotubes by changing the pressure in floating catalyst CVD," Carbon, vol. 44, pp. 18531859,2006

[13] C. Lu and J. Liu, "Controlling the Diameter of Carbon Nanotubes in Chemical Vapor Deposition Method by Carbon Feeding," $J$. Phys. Chem. B, vol. 110, pp. 20254-20257, 2006.

[14] C. L. Cheung, A. Kurtz, H. Park, and C. M. Lieber, "DiameterControlled Synthesis of Carbon Nanotubes," J. Phys. Chem. B, vol. 106, pp. 2429-2433, 2002.

[15] P. Nikolaev, M. J. Bronikowski, R. K. Bradley, F. Rohmund, D. T. Colbert, K.A. Smith, and R. E. Smalley, "Gas-phase catalytic growth of single-walled carbon nanotubes from carbon monoxide," Chemical Physics Letters, vol. 313, pp. 91-97, 1999.

[16] O. Zhou, H. Shimoda, B. Gao, S. Oh, L. Fleming, and G. Yue, "Materials Science of Carbon Nanotubes: Fabrication, Integration, and Properties of Macroscopic Structures of Carbon Nanotubes," Accounts of Chemical Research, vol. 35, pp. 1045-1053, 2002.

[17] P. Avouris, M. Freitag, and V. Perebeinos, "Carbon-nanotube photonics and optoelectronics," Nature photonics, vol. 2, pp. 341$350,2008$.

[18] J. Guo, S. Datta, and M. Lundstrom, "A Numerical Study of Scaling Issues for Schottky-Barrier Carbon Nanotube Transistor," IEEE Transactions on Electron Devices, vol. 51, pp. 172-177, 2004.

[19] A. Hazeghi, T. Krishnamohan, and H.-S. P. Wong, "SchottkyBarrier Carbon Nanotube Field-Effect Transistor Modeling," IEEE Transactions on Electron Devices, vol. 54, pp. 439-445, 2007.

[20] A. Raychowdhury, S. Mukhopadhyay, and K. Roy, "Modeling of Ballistic Carbon Nanotube Field Effect Transistors for Efficient Circuit Simulation," in International Conference on Computer Aided Design (ICCAD'03), 2003.

[21] J. Deng and H.-S. P. Wong, "A Compact SPICE Model for Carbon-Nanotube Field-Effect Transistors Including Nonidealities 
and Its Application-Part II: Full Device Model and Circuit Performance Benchmarking," IEEE Transactions on Electron Devices, vol. 54, pp. 3195-3205, 2007. S. O. Koswatta, N. Neophytou, D. Kienle, G. Fiori, and M. S. Lundstrom, "Dependence of DC Characteristics of CNT MOSFETs on Bandstructure Models," IEEE Transactions on Nanotechnology, vol. 5, pp. 368-372, 2006

[23] J. W. Mintmire and C. T. White, "Electronic and Structural properties of Carbon nanotubes," Carbon, vol. 33 pp. 893-902 1995.

[24] R. Saito, M. Fujita, G. Dresselhaus, and M. S. Dresselhaus, "Electronic structure of chiral graphene tubules," Applied Physics Letters vol. 60, pp. 2204-2206, 1992.

[25] J. W. G. Wildoer, L. C. Venema, A. G. Rinzler, R. E. Smalley, and C. Dekker, "Electronic Structure of atomically resolved carbon nanotubes," Nature, vol. 391, pp. 59-62, 1998.

[26] N. Hamada, S. Sawada, and A. Oshiyama, "New one-dimensional conductors: Graphitic microtubules " Phys. Rev. Lett., vol. 68, pp. $1579-1581,1992$

[27] R. Saito, M. S. Dresselhaus, and G. Dresselhaus, Physical Properties of Carbon Nanotubes: Imperial College Press, 1998.

[28] S. Reich, J. Maultzsch, C. Thomsen, and P. Ordejón, "Tightbinding description of graphene," Physical Review B, vol. 66, p. 035412,2002

[29] D. Kienle, J.I. Cerda, and A. W. Ghosh, "Extended Hückel theory for bandstructure, chemistry and transport : I. Carbon Nanotubes," J. Appl. Phys., vol. 100, p. 043714., 2006.

[30] O. Gülseren, T. Yildirim, and S. Ciraci, "Systematic ab initio study of curvature effects in carbon nanotubes," Physical Review $B$, vol. 65 , p. $153405,2002$.

[31] T. W. Odom, J. Huang, P. Kim, and C. M. Lieber, "Atomic structure and electronic properties of single-walled carbon nanotubes," Nature, vol. 391, pp. 62-64, 1998.

[32] S. Reich, C. Thomsen, and P. Ordejon, "Electronic band structure of isolated and bundled carbon nanotubes," Physical Review B, vol. 65 , p. 155411,2002

[33] Y. Sato, K. Yanagi, Y. Miyata, K. Suenaga, H. Kataura, and S. Iijima, "Chiral-Angle Distribution for Separated Single-Walled Carbon Nanotubes," Nano Letters, vol. 8, pp. 3151-3154, 2008.

[34] J. W. Ding, X. H. Yan, and J. X. Cao, "Analytical relation of band gaps to both chirality and diameter of single-wall carbon nanotubes," Physical Review B, vol. 66, p. 073401, 2002.

[35] J. R. Hauptmann, "Spin-Transport in Carbon Nanotubes," in Faculty of Science. vol. Master of Science: University of Copenhagen, 2003.

[36] K. El Shabrawy, K. Maharatna, D. M Bagnall, and B. M. AlHashimi, "A new analytical model for predicting SWCNT bandgap from geometrical properties," in ICICDT Grenoble, France, 2008.

[37] C. L. Kane and E. J. Mele, "Size, Shape, and Low Energy Electronic Structure of Carbon Nanotubes," Physical Review Letters, vol. 78, pp. 1932 - 1935, 1997.

[38] D. Akinwande, Y. Nishi, and H.-S. P. Wong, "An Analytical Derivation of the Density of States, Effective Mass, and Carrier Density for Achiral Carbon Nanotubes," IEEE Transactions on Electron Devices, vol. 55, pp. 289-297, 2008.

[39] S. Bandow, S. Asaka, Y. Saito, A. M. Rao, L. Grigorian, E. Richter, and P. C. Eklund, "Effect of the Growth Temperature on the Diameter Distribution and Chirality of Single-Wall Carbon Nanotubes," Physical Review Letters, vol. 80, pp. 3779-3782, 1998.

[40] S. Ahmad, M. Abdollahian, and P. Zeephongsekul, "Process Capability Analysis for Non-Normal Quality Characteristics Using Gamma Distribution," in International Conference on Information Technology (ITNG'07), 2007.

[41] C. Thomsen, S. Reich, and J. Maultzsch, Carbon Nanotubes: Basic Concepts and Physical Properties: Wiley-VCH, 2004

[42] X. Zhou, J. Park, S. Huang, J. Liu, and P. L. McEuen, "Band Structure, Phonon Scattering, and the Performance Limit of Single-Walled Carbon Nanotube Transistors," Physical Review Letters, vol. 95, p. 1468052005.
V. Perebeinos, J. Tersoff, and P. Avouris, " Electron-Phonon Interaction and Transport in Semiconducting Carbon Nanotubes," Physical Review Letters, vol. 94, p. 086802, 2005.

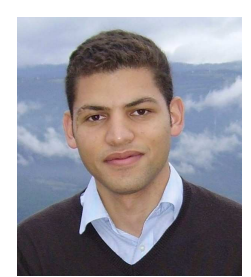

Karim El Shabrawy received a Masters of Engineering degree (Hons.) in Electrical and Electronic Engineering from the University of Bristol, UK. He is currently working toward the Ph.D degree in electronic engineering at the University of Southampton, UK. His research interests involve modeling and exploring novel applications for carbon nanotube devices. He was granted a Best Student Paper Award in the 2008 ICICDT Conference. He is presently the Chairman of the IEEE Student Branch at the University of Southampton.

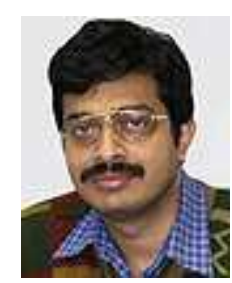

Koushik Maharatna received his M.Sc. degree in Electronics Science from Calcutta University, Calcutta, India in 1995 and Ph.D. degree from Jadavpur University, Calcutta, India, in 2002. From 1996 to 2000, he was involved in different projects sponsored by Government of India undertaken at the Indian Institute of Technology (IIT) Kharagpur, India. From 2000 to 2003, he was a Research Scientist with IHP, Frankfurt (Oder), Germany. During this period, his main involvement was related to the design of a single-chip modem for the IEEE 802.11a standard. In August 2003, he joined the Department of Electrical \& Electronics Engineering, University of Bristol, Bristol, UK as a Lecturer. From October 2006 he is with the School of Electronics and Computer Science, University of Southampton, where he is currently a Senior Lecturer. His research interests include development of VLSI architectures for the application in analogue and digital signal processing, computer arithmetic, low-power design, and nano-circuits.

$\mathrm{He}$ is currently a member of the Engineering and Physical Research Council (EPSRC) College in the UK and a member of IEEE Circuit \& Systems society's VLSI Systems Application Technical Committee (VSATC).

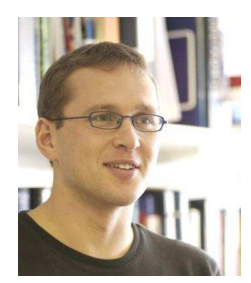

Darren Bagnall heads Photovoltaics Research within the Nano Group of the School of Electronics and Computer Science at Southampton University. His energetic research team is focussed on the exploration of nanophotonic and microelectronics concepts and their application to photovoltaics. His current work includes the use of biomimetic antireflective surfaces and plasmonic structures for enhanced absorption and enhanced scattering/lighttrapping within thin film silicon solar cells. Extensive silicon based device fabrication and lithographic capabilities available in Southampton are also enabling an exploration of innovation device structures and fabrication techniques. Prof. Bagnall completed his $\mathrm{PhD}$ on $\mathrm{CuInSe} 2$ based solar cells in the Electronics Department at Salford University. He then became a research assistant at Strathclyde University studying II-VI based blue lasers before taking up a research fellowship in Japan at the Institute of Materials Research of Tohoku University and then at the Photodynamics Research Centre, RIKEN. During this time he continued his research on short-wavelength laser and was the first to report the laser operation of $\mathrm{ZnO}$ at room temperature. His background in semiconductor deposition and optoelectronic devices brought him to Southampton in 1999, where, looking for new challenges he developed a strong interest in optical metamaterials and the combination of photonics with photovoltaics. 


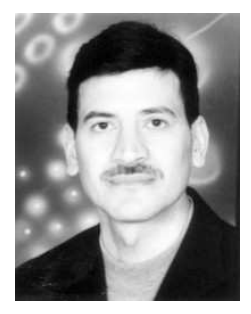

Bashir M. Al-Hashimi (M'99-SM'01-F'09) received the B.Sc. degree (with 1st-class classification) in Electrical and Electronics Engineering from the University of Bath, UK, in 1984 and the Ph.D. degree from York University, UK, in 1989. Following this he worked in the microelectronics design industry and in 1999, he joined the School of Electronics and Computer Science, Southampton University, UK, where he is currently a Full Professor of Computer Engineering and Director of the Pervasive System Center. He has authored one book on SPICE simulation, (CRC Press, 1995), and coauthored two books, Power Constrained Testing of VLSI circuits (Springer, 2002), and System-Level Design Techniques for Energy-Efficient Embedded Systems (Springer, 2004). In 2006, he edited the book, System-on-Chip: Next Generation Electronics (IEE Press, 2006). He has published over 240 papers in journals and refereed conference proceedings and supervised $25 \mathrm{PhD}$ theses successfully. His current research interests include low-power system-level design, system-on-chip test, and reliable nano design.

Prof. Al-Hashimi is a Fellow of the IEE, Fellow of the British Computer Society (BCS), and Fellow of the IEEE. He is the Editor-in-Chief of the IEE Proceedings: Computers and Digital Techniques, an editor of the Journal of Electronic Testing: Theory and Applications (JETTA), and is a member of the editorial board of the Journal of Low Power Electronics, and the Journal of Embedded Computing. He served as the General Chair of the 11th IEEE European Test Symposium (UK 2006), Technical Programme Chair of DATE 09 and will serve as General Chair DATE 2011. He is the coauthor of the James Beausang Best Paper Award at the 2000 IEEE International Test Conference relating to low power BIST for RTL data paths, and a co-author of a paper on test data compression which has was selected for a Springer book featuring the most influential work over the 10 years of the Design Automation and Test in Europe (DATE) conference. 